\title{
Measurement of cross sections in Higgs boson decays to four leptons with the ATLAS detector
}

\author{
Susumu Oda*, on behalf of the ATLAS Collaboration \\ Department of Physics, Faculty of Science, Kyushu University \\ E-mail: Susumu.Oda@cern.ch
}

\begin{abstract}
Higgs boson decays to four leptons can be selected with a very high purity and are very well suited for measurements of Higgs boson properties, despite the small branching fraction. Measurements of differential cross sections and cross sections of different Higgs boson production processes in the simplified template cross section framework using proton-proton collision data produced at the Large Hadron Collider at a center of mass energy of $13 \mathrm{TeV}$ and recorded by the ATLAS detector from 2015 to 2018 are presented.
\end{abstract}

European Physical Society Conference on High Energy Physics - EPS-HEP2019 -

10-17 July, 2019

Ghent, Belgium

${ }^{*}$ Speaker. 


\section{Introduction}

Higgs boson $(H)$ decays to four leptons via two $Z$ bosons can be selected with a very high purity and are very well suited for measurements of Higgs boson properties, despite the small branching fractions. Updated measurements of the Higgs boson properties in the $H \rightarrow Z Z^{*} \rightarrow 4 \ell$ decay channel (where $\ell=e$ or $\mu$ ) are presented using $139 \pm 2 \mathrm{fb}^{-1}$ of proton-proton ( $p p$ ) collision data collected at a center of mass energy of $\sqrt{s}=13 \mathrm{TeV}$ by the ATLAS detector [1] in the LHC Run 2 between 2015 and 2018 [2]. Main updates from previous results at $13 \mathrm{TeV}$ [3, 4, 5] include (i) full LHC Run 2 integrated luminosity, (ii) improved lepton isolation to mitigate the impact of additional $p p$ interactions in the same bunch crossing (pileup), (iii) constraint of the major non-resonant $Z Z^{*}$ background by dedicated data side-band regions, (iv) unfolding method for differential variables exploiting the full response matrix, (v) additional reconstructed event categories and new discriminants to enhance the sensitivity to the various production modes, (vi) dedicated control region to constraint the background in the categories probing the $t t H$ production $(t X X$ background), dominated by $t t Z$.

\section{Cross section measurement}

Inclusive fiducial cross section, total cross section, differential fiducial cross section and production mode cross section were measured. Differential fiducial cross section is measured as a function of the transverse momentum $\left(p_{\mathrm{T}}\right)$ of four lepton system and the number of jets to test the Standard Model (SM) prediction and constraint beyond the SM (BSM) effects because these quantities are expected to be sensitive to higher-order QCD calculations, the modeling of gluon emission, the fractions of the different production modes, the Lagrangian structure of the Higgs boson interactions. Production mode cross section is measured in the framework of Simplified Template Cross sections (STXS) framework [6]. Kinematic regions sensitive to different Higgs boson production mechanisms or production kinematic regions (production bins) are defined at the particle level for Stage $0(\mathrm{ggF}, \mathrm{VBF}, V H, t t H)$ and reduced Stage 1.1. The $b b H$ contributions are included in the ggF production bins. Corresponding reconstructed event categories are defined for signal and side-band regions. Fiducial selection follows closely reconstruction-level selection to be model independent. Unfolding is used to correct for bin migrations, detector resolution and efficiency effects.

\section{Event selection}

Template fit of four-lepton mass $\left(m_{4 \ell}\right)$ distribution is used to extract the number of signal events. The signal mass window is $115 \mathrm{GeV}<m_{4 \ell}<130 \mathrm{GeV}$, and side-band mass windows are $105 \mathrm{GeV}<m_{4 \ell}<115 \mathrm{GeV}$ and $130 \mathrm{GeV}<m_{4 \ell}<350 \mathrm{GeV}$. Table 1 shows the definition of the fiducial phase space. Matrix elements are used for extra leptons in the case of $V H$ leptonic and $t t H$ leptonic production modes to avoid mis-pairing.

Discriminants of structured neural networks are exploited to separate processes and to improve sensitivities. Inputs of a multilayer perceptron (MLP) are a recurrent neural network (rNN) for the vectors of $p_{\mathrm{T}}$ and $\eta$ for four lepton system, another rNN for up to three jets and another MLP with additional variables. 


\begin{tabular}{lc}
\hline \hline & Leptons and jets \\
Leptons & $p_{\mathrm{T}}>5 \mathrm{GeV},|\eta|<2.7$ \\
Jets & $p_{\mathrm{T}}>30 \mathrm{GeV},|y|<4.4$ \\
Remove jets with & $\Delta R($ jet,$\ell)<0.1$ \\
\hline & Lepton selection and pairing \\
Lepton kinematics & $p_{\mathrm{T}}>20,15,10 \mathrm{GeV}$ \\
Leading pair $\left(m_{12}\right)$ & SFOS lepton pair with smallest $\left|m_{Z}-m_{\ell \ell}\right|$ \\
Sub-leading pair $\left(m_{34}\right)$ & Remaining SFOS lepton pair with smallest $\left|m_{Z}-m_{\ell \ell}\right|$ \\
\hline \multicolumn{3}{c}{ Event selection (at most one quadruplet per event) } \\
Mass requirements & $50 \mathrm{GeV}<m_{12}<106 \mathrm{GeV}$ and $12 \mathrm{GeV}<m_{34}<115 \mathrm{GeV}$ \\
Lepton separation & $\Delta R\left(\ell_{i}, \ell_{j}\right)>0.1$ \\
$J / \psi$ veto & $m\left(\ell_{i}, \ell_{j}\right)>5 \mathrm{GeV}$ for all SFOS lepton pairs \\
Mass window & $105 \mathrm{GeV}<m_{4 \ell}<160 \mathrm{GeV}$ \\
If extra leptons with $p_{\mathrm{T}}>12 \mathrm{GeV}$ & Quadruplet with the largest matrix element \\
\hline \hline
\end{tabular}

Table 1: List of event selection requirements which define the fiducial phase space for the cross section measurement. SFOS lepton pairs are same-flavor opposite-sign lepton pairs [2].

\section{Systematic uncertainties}

\begin{tabular}{ccccc}
\hline \hline Production mode & $\mathrm{ggF}$ & $\mathrm{VBF}$ & $\mathrm{VH}$ & $\mathrm{ttH}$ \\
\hline Luminosity & $1.7 \%$ & $1.7 \%$ & $1.8 \%$ & $1.7 \%$ \\
$e, \mu$, pileup & $1.5 \%$ & $1 \%$ & $1.5 \%$ & $1 \%$ \\
Jets, flavor tagging & $1 \%$ & $4.5 \%$ & $3.5 \%$ & $4.5 \%$ \\
Reducible background & $0.5 \%$ & $0.5 \%$ & $1 \%$ & $1 \%$ \\
\hline$Z Z^{*}$ background & $1.5 \%$ & $2 \%$ & $5 \%$ & $1 \%$ \\
$t X X$ background & $<0.5 \%$ & $0.5 \%$ & $0.5 \%$ & $0.5 \%$ \\
PDF & $0.5 \%$ & $1.5 \%$ & $2 \%$ & $0.5 \%$ \\
QCD scale & $1 \%$ & $8 \%$ & $12 \%$ & $8 \%$ \\
Signal parton shower & $2 \%$ & $6 \%$ & $8 \%$ & $4 \%$ \\
\hline \hline
\end{tabular}

Table 2: The impact of the dominant systematic uncertainties on the Stage 0 production mode cross sections [2].

Table 2 shows systematic uncertainties on the Stage 0 production mode cross sections. Many of systematic uncertainties have been decreased from previous results. Luminosity uncertainty was reduced to $1.7 \%$ by a new calibration. Electron and muon reconstruction and identification efficiencies and their pileup dependence had dominant uncertainties. Those were reduced to about $50 \%$ of previous results. The side-band regions were introduced for the $Z Z Z^{*}$ background and the uncertainty was reduced to about $60 \%$ of previous results. 


\section{Results}

Figure 1 shows the inclusive $m_{4 \ell}$ distributions. The $H \rightarrow 4 \ell$ and $Z \rightarrow 4 \ell$ peaks are clearly seen. Although the expected distribution is pre-fit one, good agreement between the observation and expectation is seen. In the mass window of $115 \mathrm{GeV}<m_{4 \ell}<130 \mathrm{GeV}, 316$ events were observed.

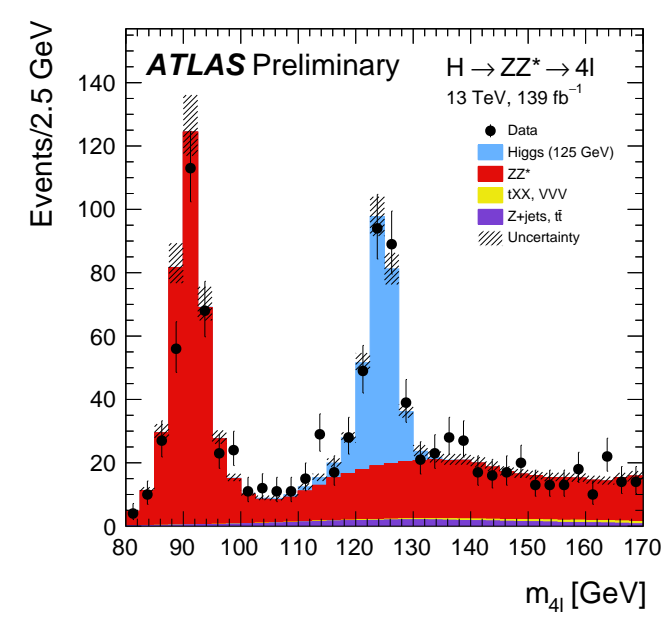

Figure 1: The observed and expected (pre-fit) inclusive four-lepton invariant mass distributions [2].

Figure 2 shows NN discriminants, which separate different production modes, and signal and backgrounds. In the $0 j-p_{\mathrm{T}}^{4 \ell}$-Med category, the discriminant is used to separate $\mathrm{ggF}$ signal and $\mathrm{ZZ}^{*}$ background. In the $2 j$ category, the discriminant is used to separate $\mathrm{ggF}$ and VBF signals. Good agreement between observation and expectation is seen.
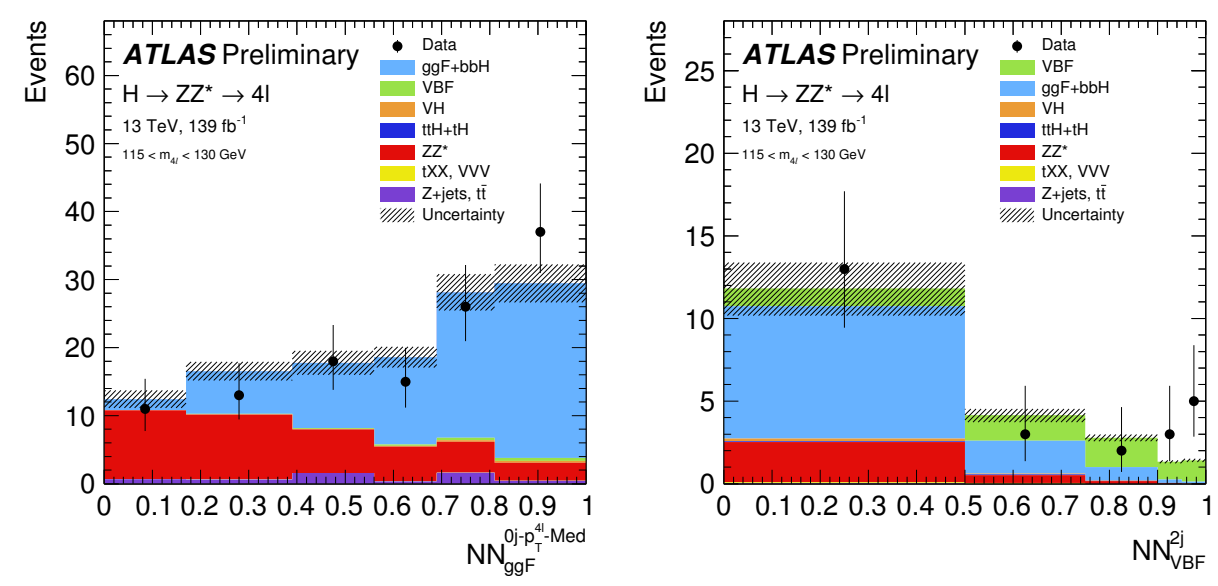

Figure 2: The observed and expected $\mathrm{NN}$ output (pre-fit) distributions in $\mathrm{NN}_{\mathrm{ggF}}$ in $0 j-p_{\mathrm{T}}^{4 \ell}$-Med (left) and $\mathrm{NN}_{\mathrm{VBF}}$ in $2 j$ with $\mathrm{NN}_{\mathrm{VH}}<0.2$ (right) [2]. 


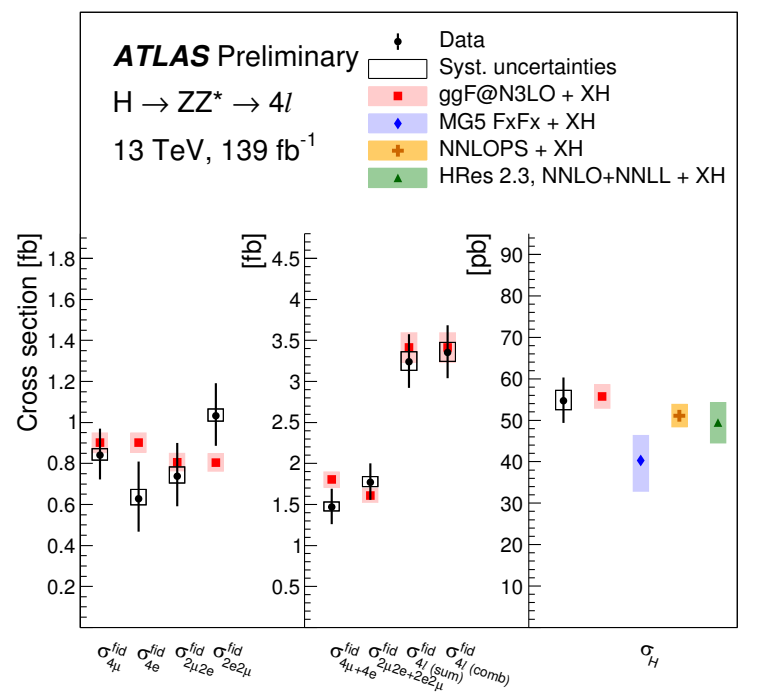

Figure 3: The fiducial cross section (left two panels) and total cross section (right panel) of Higgs boson production measured in the $4 \ell$ final state [2].

Figure 3 shows the fiducial cross sections (left two panels) and total cross section (right panel). The fiducial cross sections are shown separately for each decay final state, and for same- and opposite-flavor decays. The inclusive fiducial cross section is measured as the sum of all final states, as well as by combining the per-final state measurements assuming SM $Z Z^{*} \rightarrow 4 \ell$ relative branching fractions. The total cross section is compared to the cross section predictions by $\mathrm{N}^{3} \mathrm{LO}$, MadGraph5_aMC@NLO with FxFx merging, NNLOPS and HRes for ggF production mode. The $p$-values indicating the compatibility of the measurement and the SM prediction with $\mathrm{N}^{3} \mathrm{LO}$ range from $11 \%$ to $85 \%$.

Figure 4 shows differential cross sections as a function of $p_{\mathrm{T}}$ of four lepton system and the number of jets. All samples are normalized to the most accurate SM predictions. Fitted values of $Z Z^{*}$ background normalization factors are shown in the bottom panels. Good agreement between data and predictions by MadGraph5_aMC@NLO with FxFx merging and NNLOPS with $K$ factors is seen.

The inclusive $H \rightarrow Z Z^{*}$ production cross section times branching fraction $(\sigma \cdot \mathscr{B})$ for $\left|y_{H}\right|<2.5$ is measured to be

$$
\sigma \cdot \mathscr{B}=1.38 \pm 0.11 \text { (statistical) }{ }_{-0.03}^{+0.05}(\text { experimental }) \pm 0.03 \text { (theoretical) } \mathrm{pb}=1.38 \pm 0.12 \mathrm{pb} \text {. }
$$

The $\mathrm{SM}$ prediction is $(\sigma \cdot \mathscr{B})_{\mathrm{SM}}=1.33 \pm 0.09 \mathrm{pb}$. The global signal strength is

$$
\left.\mu=1.04_{-0.08}^{+0.09}(\text { statistical })_{-0.03}^{+0.04}(\text { experimental })_{-0.05}^{+0.06} \text { (theoretical }\right)=1.04_{-0.10}^{+0.12}
$$

and is consistent with the SM prediction with a $p$-value of $71 \%$.

Ratios of the observed $\sigma \cdot \mathscr{B}$ to that predicted by the SM for Stage 0 and reduced Stage 1.1 are shown in Figure 5. All Stage 0 and reduced Stage $1.1 \mathrm{ggF}$ measurements agree with the predictions for the SM Higgs boson within 1.5 standard deviation. 

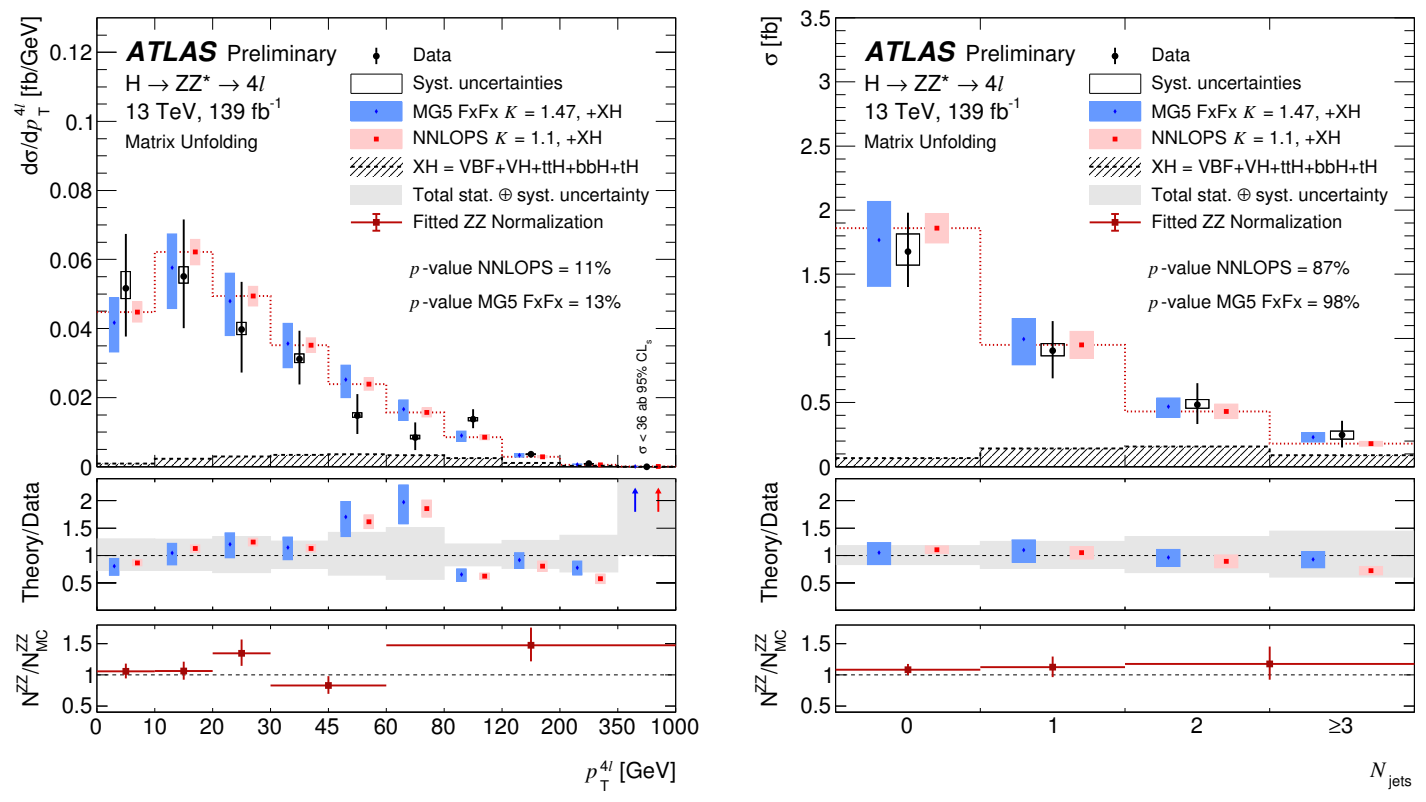

Figure 4: Differential fiducial cross sections for the transverse momentum $p_{\mathrm{T}}^{4 \ell}$ of the Higgs boson (left) and the number of jets $N_{\text {jets }}$ (right) [2].

\section{Summary}

With the full Run 2 statistics, inclusive fiducial, differential fiducial and production mode cross section measurements of Higgs boson decays to four leptons are presented. All measurements are consistent with the Standard Model predictions.

\section{Acknowledgment}

This work was supported in part by MEXT KAKENHI Grant Number 16H06491, JSPS KAKENHI Grant Number JP26800140 and the Progress 100 program of Kyushu University.

\section{References}

[1] ATLAS Collaboration, The ATLAS Experiment at the CERN Large Hadron Collider, JINST 3 (2008) S08003.

[2] ATLAS Collaboration, Measurements of the Higgs boson inclusive, differential and production cross sections in the $4 \ell$ decay channel at $\sqrt{s}=13 \mathrm{TeV}$ with the ATLAS detector, ATLAS-CONF-2019-025, 2019, http: / / cdsweb. cern. ch/record/2682107.

[3] ATLAS Collaboration, Measurement of inclusive and differential cross sections in the $H \rightarrow Z Z^{*} \rightarrow 4 \ell$ decay channel in pp collisions at $\sqrt{s}=13 \mathrm{TeV}$ with the ATLAS detector, JHEP 10 (2017) 132.

[4] ATLAS Collaboration, Measurement of the Higgs boson coupling properties in the $H \rightarrow Z Z^{*} \rightarrow 4 \ell$ decay channel at $\sqrt{s}=13$ TeV with the ATLAS detector, JHEP 03 (2018) 095. 

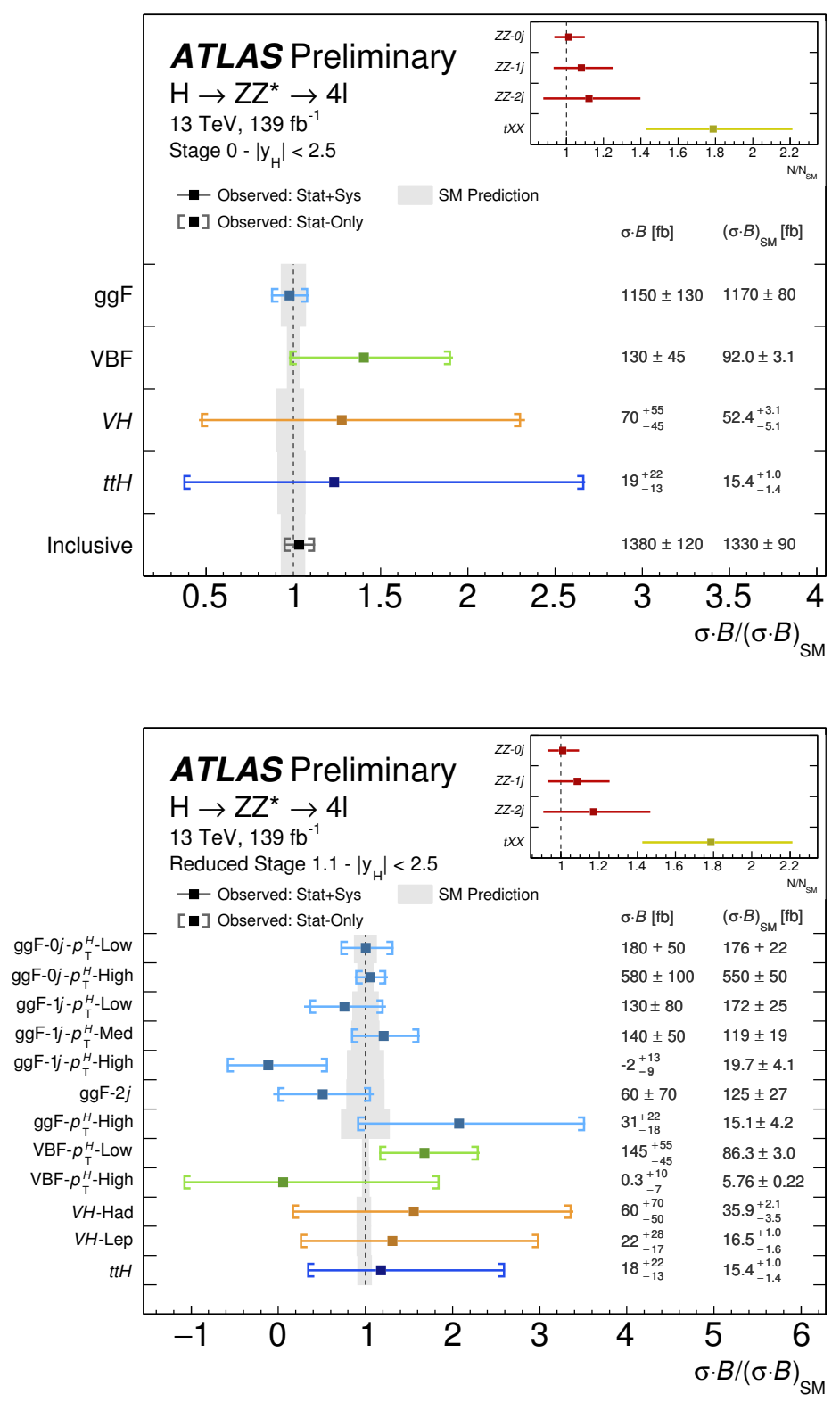

Figure 5: The observed cross sections time branching fractions $\sigma \cdot \mathscr{B}$ normalized by the SM expectation $(\sigma \cdot \mathscr{B})_{\mathrm{SM}}$ for the inclusive production and in the Stage 0 (top) and the reduced Stage 1.1 production bins (bottom) [2].

[5] ATLAS Collaboration, Measurements of the Higgs boson production, fiducial and differential cross sections in the $4 \ell$ decay channel at $\sqrt{s}=13 \mathrm{TeV}$ with the ATLAS detector, ATLAS-CONF-2018-018, 2018, http: //cds.cern. ch/record/2621479.

[6] N. Berger et al., Simplified Template Cross Sections - Stage 1.1, 2019, arXiv: 1906.02754 [hep-ph]. 